Spectroscopic data for atomic tungsten transitions of interest in fusion plasma research

This article has been downloaded from IOPscience. Please scroll down to see the full text article.

2011 J. Phys. B: At. Mol. Opt. Phys. 44145005

(http://iopscience.iop.org/0953-4075/44/14/145005)

View the table of contents for this issue, or go to the journal homepage for more

Download details:

IP Address: 91.178.108.30

The article was downloaded on 18/07/2011 at 10:57

Please note that terms and conditions apply. 


\title{
Spectroscopic data for atomic tungsten transitions of interest in fusion plasma research
}

\author{
P Quinet $^{1,2}$, P Palmeri ${ }^{1}$ and É Biémont ${ }^{1,2}$ \\ ${ }^{1}$ Astrophysique et Spectroscopie, Université de Mons-UMONS, B-7000 Mons, \\ Belgium \\ 2 IPNAS, Université de Liège, B15 Sart Tilman, B-4000 Liège, Belgium \\ E-mail: quinet@umons.ac.be
}

Received 26 April 2011, in final form 7 June 2011

Published 6 July 2011

Online at stacks.iop.org/JPhysB/44/145005

\begin{abstract}
Transition probabilities for W I lines of potential interest for fusion plasma diagnostics are reported in this paper. They have been obtained using the relativistic Hartree-Fock approach including core-polarization effects. The accuracy of the results has been assessed through comparison between two different physical models and through detailed comparison with available experimental lifetimes and transition probabilities. These results represent the first complete set of spectroscopic parameters computed for a very complex tungsten atomic system. The new data are expected to be useful for plasma modelling in fusion reactors such as the International Thermonuclear Experimental Reactor.
\end{abstract}

\section{Introduction}

Reliable spectroscopic parameters in the tungsten atom are of fundamental importance for the study and modelling of plasmas in fusion reactors. Indeed, tungsten is a very attractive element to be used as a plasma-facing material in tokamak devices because of its high melting point $\left(3410^{\circ} \mathrm{C}\right)$ and thermal conductivity, its low tritium retention and erosion rate under plasma loading (see e.g. Federici et al 2001, Pospieszczyk 2006). Some years ago, several experiments were carried out in the ASDEX Upgrade device to prove the suitability of $\mathrm{W}$ as a divertor target material under the conditions of a high-density and low-temperature divertor (Naujoks et al 1996). The International Thermonuclear Experimental Reactor (ITER) will be the next step experimental fusion device which will use tungsten, together with beryllium and carbon-fibre reinforced composites, as plasma-facing materials. The main disadvantage of tungsten in these conditions is the large radiative loss due to plasma contamination and related to its high radiative efficiency.

In fusion reactors, tungsten will be sputtered from the plasma wall as a neutral element and the intensity of the well-known W I emission line at $400.8753 \mathrm{~nm}$ may be used to estimate tungsten influx (Skinner 2008). Unfortunately, a complication arises from the fact that a coincident W II line exists at $400.8751 \mathrm{~nm}$.

The determination of the tungsten influx rate to the core plasma will depend on a calculation of transport from the wall surface through the scrape-off layer. Consequently, the identification of emission lines from the lowest ionization stages of tungsten, including the neutral atom, will greatly aid modelling of the plasma edge and scrape-off layer transport and facilitate the analysis of net tungsten influx rates.

Up to now, transition probabilities in neutral tungsten were essentially obtained experimentally by Den Hartog et al (1987) and Kling and Kock (1999) for a selection of lines depopulating energy levels for which radiative lifetimes had been measured in laboratory. The aim of this paper is to extend the set of available transition rates for W I lines not considered so far. The method used is based on the relativistic HartreeFock (HFR) approach including core-polarization corrections. This work is an extension of similar investigations carried out in W II and W III ions (Nilsson et al 2008, Palmeri et al 2008). Furthermore, let us note that critically evaluated transition rates available in the literature for allowed electric dipole transitions together with a new set of computed $A$-values for forbidden lines were reported in our recent paper (Quinet et al 2010) for W I, W II and W III. 


\section{Available spectroscopic data in W I}

\subsection{Wavelengths and energy levels}

Wavelengths of all the transitions observed in the W I spectrum, and energy levels derived from these wavelengths, were compiled by Kramida and Shirai (2006) who critically evaluated the data published previously by Laun and Corliss (1968), Shadmi and Caspi (1968), Corliss (1969), Wyart (1978), Martin et al (1978) and Campbell-Miller and Simard (1996). Of the 6800 arc lines listed by Kramida and Shirai (2006) in the spectral region 200-1048 nm, about 5500 were classified using 101 even-parity levels in the range $0-56833 \mathrm{~cm}^{-1}$ and 365 odd-parity levels in the range 19389$63533 \mathrm{~cm}^{-1}$. Whereas the 70 lowest even-parity levels belonging to the $5 d^{4} 6 s^{2}$ and $5 d^{5} 6 s$ configurations were studied parametrically by Shadmi and Caspi (1968), the system of odd-parity levels had not received adequate interpretation until the recent investigation by Wyart (2010). In this latter work, a comprehensive study of all odd-parity levels with energies lower than $45000 \mathrm{~cm}^{-1}$ was performed using the HFR method supported by the semi-empirical fitting of radial parameters corresponding to the $5 d^{4} 6 s 6 p, 5 d^{5} 6 p$ and $5 d^{3} 6 s^{2} 6 p$ configurations. This led to the classification of the 141 lowest odd-parity levels including two new levels located at $29823.09 \mathrm{~cm}^{-1}(J=0)$ and $37451.33 \mathrm{~cm}^{-1}(J=6)$ that were the only odd-parity levels still missing below $43000 \mathrm{~cm}^{-1}$.

\subsection{Radiative lifetimes and transition probabilities}

Transition probabilities in neutral tungsten were first determined experimentally by Corliss and Bozman (1962) but their arc measurements later on were recognized to be affected by large systematic errors. These results were supplemented by the relative measurements of Clawson and Miller (1973) and by the absolute measurements of Obbarius and Kock (1982). In the latter case, a stabilized arc, operated in argon, was used for measuring oscillator strengths for $43 \mathrm{~W}$ I lines in the wavelength range $240-560 \mathrm{~nm}$. The accuracy of the relative data of Clawson and Miller was estimated to range between 6 and $50 \%$ while the $f$-values measured by Obbarius and Kock were reported with uncertainties from 10 to $36 \%$.

The first experimental measurements of radiative lifetimes in W I were performed by Duquette et al (1981) who used time-resolved laser-induced fluorescence (TR-LIF) and a hollow cathode effusive atomic beam source for lifetime determination of 15 levels belonging to the $5 d^{4} 6 s 6 p$ and $5 \mathrm{~d}^{5} 6 \mathrm{p}$ configurations with an accuracy of $\pm 5 \%$. Kwiatkowski et al (1982) reported lifetimes for 13 energy levels in the configurations $(5 d+6 s)^{5} 6 \mathrm{p}$. These measurements were based on the observation of the remitted fluorescence with the singlephoton-counting technique after a selective excitation of an atomic beam by a pulsed dye laser. Some radiative lifetimes of W I excited states were also published by Plekhotkin and Verolainen (1985).

The TR-LIF technique was also used by Schnabel and Kock (1997) to measure radiative lifetimes for 47 W I levels in the energy range 27800 to $48200 \mathrm{~cm}^{-1}$ with an accuracy of $2-7 \%$. These results agree, within the mutual uncertainties, with the measurements (accurate to $\pm 5 \%$ ) of Den Hartog et al (1987) (three lifetimes common to both works). The lifetimes of Kwiatkowski et al agree within 7\% with those of Den Hartog et al (1987) (13 levels in common), the latter work including remeasurements of the values published by Duquette et al (1981).

The most extensive sets of experimental transition probabilities in neutral tungsten were reported by Den Hartog et al (1987) and Kling and Kock (1999) who measured branching fractions (BF) on high-resolution Fourier transform spectra and were able to deduce absolute transition probabilities for a set of 572 lines covering the wavelength range 225-1035 nm and involving excited energy levels up to $46932 \mathrm{~cm}^{-1}$. The typical uncertainties of $A$-values for the most intense lines $\left(A \geqslant 10^{6} \mathrm{~s}^{-1}\right)$ were estimated to range between 5 and $20 \%$, and between 7 and $9 \%$ for the former and the latter authors, respectively. The lifetimes used in these two papers were those of Den Hartog et al (1987) and of Schnabel and Kock (1997), respectively. As the work of Kling and Kock (1999) was focused on higher lying levels than that of Den Hartog et al (1987), the overlap of the two works is small. In fact, BFs were measured for only 19 lines in common. The agreement is within a few $(<7) \%$ for the most intense transitions $(\mathrm{BF}>20 \%)$, larger discrepancies appearing (as expected) for some weaker lines.

More recently, Wyart (2010) used the HFR method of Cowan (1981) for generating a set of computed transition probabilities for a selection of 70 lines depopulating 12 oddparity levels. Although obtained by considering a rather simple physical model (including only $5 d^{4} 6 s^{2}+5 d^{5} 6 s+5 d^{6}$ and $5 d^{4} 6 s 6 p+5 d^{5} 6 p+5 d^{3} 6 s^{2} 6 p$ for even and odd parities, respectively), fair qualitative agreement was noted between these calculated $g A$-values and the observed line intensities reported in the compilation of Kramida and Shirai (2006).

\section{Computational method}

The theoretical method considered for computing the decay rates in W I is the well-established HFR approach originally developed by Cowan (1981) in which we have included corepolarization effects (see e.g. Quinet et al 1999). Two sets of calculations were performed in this work. In the first one (model A), the following configurations were explicitly included: $5 \mathrm{~d}^{4} 6 \mathrm{~s}^{2}, 5 \mathrm{~d}^{5} 6 \mathrm{~s}, 5 \mathrm{~d}^{5} 7 \mathrm{~s}, 5 \mathrm{~d}^{6}, 5 \mathrm{~d}^{4} 6 \mathrm{~s} 7 \mathrm{~s}, 5 \mathrm{~d}^{4} 6 \mathrm{~s} 6 \mathrm{~d}$, $5 d^{5} 6 \mathrm{~d}, 5 \mathrm{~d}^{4} 6 \mathrm{p}^{2}, 5 \mathrm{~d}^{4} 6 \mathrm{~d}^{2}, 5 \mathrm{~d}^{3} 6 \mathrm{~s} 6 \mathrm{p}^{2}, 5 \mathrm{~d}^{2} 6 \mathrm{~s}^{2} 6 \mathrm{p}^{2}$ (even parity) and $5 d^{4} 6 s 6 p, 5 d^{4} 6 s 7 p, 5 d^{5} 6 p, 5 d^{5} 7 p, 5 d^{4} 6 s 5 f, 5 d^{5} 5 f, 5 d^{3} 6 s^{2} 6 p$, $5 \mathrm{~d}^{3} 6 \mathrm{p}^{3}, 5 \mathrm{~d}^{2} 6 \mathrm{~s} 6 \mathrm{p}^{3}$ (odd parity). For the dipole polarizability, $\alpha_{d}$, we have adopted the value of $4.59 \mathrm{a}_{0}^{3}$ (Fraga et al 1976) corresponding to the ytterbium-like $\mathrm{W}^{4+}$ ionic core. The cut-off radius, $r_{c}$, was chosen equal to $1.99 \mathrm{a}_{0}$ which corresponds to the HFR average value $\langle r\rangle$ for the outermost $5 \mathrm{~d}$ core orbital. In the second physical model (model B), configuration interaction was considered by including the same configurations as those considered in model A except $5 d^{2} 6 s^{2} 6 p$ (even parity) and $5 \mathrm{~d}^{2} 6 \mathrm{~s} 6 \mathrm{p}^{3}$ (odd parity). In this case, the corepolarization effects corresponding to a lutecium-like ionic core were considered using the dipole polarizability of $\mathrm{W}^{3+}$, i.e. $\alpha_{d}=6.88 \mathrm{a}_{0}^{3}$ (Fraga et al 1976) and the same value as the 
Table 1. Experimental and calculated energies, Landé factors and radiative lifetimes for W I even-parity levels below $37500 \mathrm{~cm}^{-1}$.

\begin{tabular}{|c|c|c|c|c|c|c|c|c|}
\hline$J$ & $\begin{array}{l}E_{\exp }{ }^{\mathrm{a}} \\
\left(\mathrm{cm}^{-1}\right)\end{array}$ & $\begin{array}{l}E_{A}^{\mathrm{b}} \\
\left(\mathrm{cm}^{-1}\right)\end{array}$ & $\begin{array}{l}\Delta E_{A}^{\mathrm{c}} \\
\left(\mathrm{cm}^{-1}\right)\end{array}$ & $\begin{array}{l}E_{B}{ }^{\mathrm{d}} \\
\left(\mathrm{cm}^{-1}\right)\end{array}$ & $\begin{array}{l}\Delta E_{B}{ }^{\mathrm{e}} \\
\left(\mathrm{cm}^{-1}\right)\end{array}$ & $g_{\exp }{ }^{a}$ & $g_{A}^{\mathrm{b}}$ & $g_{B}{ }^{\mathrm{d}}$ \\
\hline 0 & 0.00 & -88 & 88 & -90 & 90 & & & \\
\hline 1 & 1670.29 & 1697 & -27 & 1697 & -27 & 1.51 & 1.50 & 1.50 \\
\hline 3 & 2951.29 & 2893 & 58 & 2886 & 65 & 1.98 & 1.98 & 1.98 \\
\hline 2 & 3525.53 & 3381 & -56 & 3381 & -56 & 1.48 & 1.49 & 1.49 \\
\hline 3 & 4830.00 & 4838 & -8 & 4838 & -8 & 1.50 & 1.48 & 1.48 \\
\hline 4 & 6219.33 & 6118 & 101 & 6117 & 102 & 1.49 & 1.45 & 1.45 \\
\hline 0 & 9528.06 & 9524 & 4 & 9523 & 5 & & & \\
\hline 4 & 12161.96 & 12161 & 1 & 12156 & 6 & 0.99 & 0.99 & 0.99 \\
\hline 1 & 13307.10 & 13412 & -105 & 13431 & -124 & 1.32 & 1.29 & 1.29 \\
\hline 3 & 13348.56 & 13417 & -68 & 13410 & -61 & 0.92 & 0.94 & 0.94 \\
\hline 2 & 13777.71 & 13699 & 79 & 13697 & 81 & 1.09 & 1.07 & 1.07 \\
\hline 2 & 14976.18 & 15033 & -57 & 15050 & -74 & 1.06 & 0.96 & 0.96 \\
\hline 5 & 15069.93 & 15094 & -24 & 15094 & -24 & 1.05 & 1.10 & 1.10 \\
\hline 3 & 15460.01 & 15400 & 60 & 15394 & 66 & 1.17 & 1.26 & 1.26 \\
\hline 4 & 16431.31 & 16526 & -95 & 16514 & -83 & 1.02 & 1.01 & 1.01 \\
\hline 6 & 17008.50 & 16988 & 21 & 16996 & 13 & 1.4 & 1.14 & 1.14 \\
\hline 4 & 17107.01 & 17043 & 64 & 17044 & 63 & 1.19 & 1.12 & 1.12 \\
\hline 3 & 17701.18 & 17709 & -8 & 17711 & -10 & 1.02 & 1.03 & 1.03 \\
\hline 1 & 18082.83 & 18090 & -7 & 18082 & 1 & 0.7 & 0.79 & 0.79 \\
\hline 2 & 18116.84 & 18171 & -54 & 18165 & -48 & 1.08 & 1.42 & 1.52 \\
\hline 2 & 18280.48 & 18308 & -28 & 18313 & -33 & 1.43 & 1.06 & 0.97 \\
\hline 3 & 18974.51 & 19009 & -34 & 19009 & -34 & 1.06 & 1.07 & 1.08 \\
\hline 2 & 19253.56 & 19291 & -37 & 19304 & -50 & 1.18 & 1.23 & 1.24 \\
\hline 4 & 19256.24 & 19293 & -37 & 19302 & -46 & 1.20 & 1.18 & 1.18 \\
\hline 5 & 19535.01 & 19623 & -88 & 19622 & -87 & 1.21 & 1.15 & 1.14 \\
\hline 6 & 19648.54 & 19657 & -8 & 19665 & -16 & 1.32 & 1.32 & 1.32 \\
\hline 5 & 19826.04 & 19756 & 70 & 19762 & 64 & 1.20 & 1.26 & 1.26 \\
\hline 3 & 19827.68 & 19812 & 16 & 19808 & 20 & 1.28 & 1.42 & 1.42 \\
\hline 0 & 20174.20 & 20084 & 90 & 20052 & 122 & & & \\
\hline 1 & 20427.84 & 20429 & -1 & 20423 & 5 & 2.1 & 2.11 & 2.11 \\
\hline 2 & 20983.02 & 21036 & -53 & 21041 & -58 & & 1.57 & 1.57 \\
\hline 4 & 22476.68 & 22464 & 13 & 22468 & 9 & 1.48 & 1.39 & 1.38 \\
\hline 0 & 22773.78 & 22819 & -45 & 22819 & -45 & & & \\
\hline 4 & 22852.80 & 22787 & 66 & 22772 & 81 & 1.2 & 1.11 & 1.11 \\
\hline 1 & 23455.02 & 23490 & -35 & 23488 & -33 & & 1.72 & 1.72 \\
\hline 6 & 23484.78 & 23444 & 41 & 23449 & 36 & & 1.03 & 1.03 \\
\hline 3 & 23930.08 & 23864 & 66 & 23861 & 69 & 1.4 & 1.49 & 1.49 \\
\hline 2 & 23982.80 & 23981 & 2 & 23978 & 5 & & 1.56 & 1.56 \\
\hline 3 & 24610.88 & 24628 & -17 & 24633 & -22 & & 1.03 & 1.03 \\
\hline 2 & 24789.66 & 24841 & -51 & 24860 & -70 & & 1.14 & 1.14 \\
\hline 2 & 26861.64 & 26859 & 3 & 26865 & -3 & & 0.98 & 0.98 \\
\hline 4 & 27213.82 & 27112 & 102 & 27122 & 92 & & 1.25 & 1.25 \\
\hline 1 & 27670.48 & 27816 & -146 & 27809 & -139 & & 0.15 & 0.16 \\
\hline 5 & 27849.80 & 27806 & 44 & 27804 & 46 & & 0.92 & 0.92 \\
\hline 2 & 28204.20 & 28292 & -88 & 28289 & -85 & & 1.03 & 1.03 \\
\hline 5 & 28233.44 & 28072 & 161 & 28080 & 153 & & 1.35 & 1.35 \\
\hline 3 & 28291.88 & 28229 & 63 & 28236 & 56 & & 1.11 & 1.11 \\
\hline 3 & 28347.60 & 28344 & 4 & 28336 & 12 & & 1.15 & 1.16 \\
\hline 6 & 28392.70 & 28475 & -82 & 28472 & -79 & & 1.05 & 1.05 \\
\hline 1 & 28720.88 & 28801 & -80 & 28808 & -87 & & 1.44 & 1.44 \\
\hline 2 & 28898.96 & 28814 & 85 & 28835 & 64 & & 1.15 & 1.14 \\
\hline 0 & & 29036 & & 29040 & & & & \\
\hline 3 & 29430.50 & 29520 & -89 & 29519 & -88 & & 1.00 & 1.01 \\
\hline 7 & 29460.98 & 29575 & -114 & 29574 & -113 & & 1.14 & 1.14 \\
\hline 4 & 29479.32 & 29517 & -38 & 29515 & -36 & & 1.28 & 1.28 \\
\hline 4 & 29853.66 & 29837 & 17 & 29837 & 17 & & 1.03 & 1.03 \\
\hline 2 & 30374.20 & 30275 & 99 & 30242 & 132 & & 1.12 & 1.12 \\
\hline 2 & 31077.80 & 31180 & -102 & 31190 & -112 & & 1.06 & 1.06 \\
\hline 5 & 31389.08 & 31300 & 89 & 31305 & 84 & & 1.12 & 1.12 \\
\hline 4 & 32135.94 & 32086 & 50 & 32081 & 55 & & 1.06 & 1.06 \\
\hline 3 & 32217.91 & 32236 & -18 & 32226 & -8 & & 1.04 & 1.04 \\
\hline 1 & 32378.40 & 32433 & -55 & 32436 & -58 & & 0.49 & 0.49 \\
\hline 3 & 32826.63 & 32797 & 30 & 32806 & 21 & & 1.01 & 1.00 \\
\hline 1 & & 33035 & & 33008 & & & 0.88 & 0.88 \\
\hline
\end{tabular}


Table 1. (Continued.)

\begin{tabular}{|c|c|c|c|c|c|c|c|c|}
\hline$J$ & $\begin{array}{c}E_{\exp }{ }^{\mathrm{a}} \\
\left(\mathrm{cm}^{-1}\right)\end{array}$ & $\begin{array}{l}E_{A}{ }^{\mathrm{b}} \\
\left(\mathrm{cm}^{-1}\right)\end{array}$ & $\begin{array}{l}\Delta E_{A}{ }^{\mathrm{c}} \\
\left(\mathrm{cm}^{-1}\right)\end{array}$ & $\begin{array}{l}E_{B}{ }^{\mathrm{d}} \\
\left(\mathrm{cm}^{-1}\right)\end{array}$ & $\begin{array}{l}\Delta E_{B}{ }^{\mathrm{e}} \\
\left(\mathrm{cm}^{-1}\right)\end{array}$ & $g_{\exp }{ }^{a}$ & $g_{A}{ }^{\mathrm{b}}$ & $g_{B}{ }^{\mathrm{d}}$ \\
\hline 5 & 33201.61 & 33210 & -8 & 33201 & 1 & & 1.14 & 1.14 \\
\hline 6 & 33291.80 & 33220 & 72 & 33211 & 81 & & 1.07 & 1.07 \\
\hline 4 & 33569.53 & 33589 & -20 & 33580 & -10 & & 1.01 & 1.01 \\
\hline 3 & 33952.85 & 33774 & 179 & 33775 & 178 & & 1.19 & 1.19 \\
\hline 4 & 34302.04 & 34284 & 18 & 34280 & 22 & & 1.17 & 1.16 \\
\hline 3 & 34465.83 & 34693 & -227 & 34696 & -230 & & 1.03 & 1.03 \\
\hline 2 & & 34509 & & 34508 & & & 0.96 & 0.96 \\
\hline 4 & 35299.82 & 35162 & 138 & 35168 & 132 & & 0.98 & 0.98 \\
\hline 2 & & 35504 & & 35468 & & & 1.05 & 1.05 \\
\hline 2 & & 35790 & & 35787 & & & 0.92 & 0.92 \\
\hline 0 & & 35856 & & 35807 & & & & \\
\hline 1 & & 36301 & & 36271 & & & 1.11 & 1.10 \\
\hline 5 & & 36392 & & 36392 & & & 1.12 & 1.12 \\
\hline 6 & & 37102 & & 37088 & & & 1.08 & 1.08 \\
\hline 4 & & 37284 & & 37234 & & & 1.24 & 1.24 \\
\hline 3 & 37414.11 & 37397 & 17 & 37402 & 12 & & 1.08 & 1.08 \\
\hline 2 & & 37473 & & 37480 & & & 1.06 & 1.06 \\
\hline
\end{tabular}

${ }^{a}$ From Kramida and Shirai (2006).

b Present work-model A.

c $\Delta E_{A}=E_{\text {exp }}-E_{A}$.

d Present work-model B.

e $\Delta E_{B}=E_{\exp }-E_{B}$.

one used in model A for the cut-off radius, i.e. $r_{c}=1.99 \mathrm{a}_{0}$. The only difference between both models thus lies in the way core-valence interactions are taken into account. By assuming an ionic core with 70 electrons in the core polarization potential and opening the $5 \mathrm{~d}$ subshell up to $5 \mathrm{~d}^{2}$, these interactions are considered more explicitly in model A than in model B where a slightly bigger ionic core containing 71 electrons is incorporated in the potential.

In each of these approaches, the final wavefunctions were obtained by a parametric fit of the calculated energy levels to the experimental ones. All even experimental levels compiled by Kramida and Shirai (2006) for $5 d^{4} 6 s^{2}$ and $5 d^{5} 6 s$ configurations were fitted using, as adjustable parameters, the average energies $\left(E_{\mathrm{av}}\right)$, the Slater radial integrals $\left(F^{k}, G^{k}, R^{k}\right)$, the spin-orbit parameters $\left(\zeta_{n l}\right)$ and the effective interaction parameters $(\alpha, \beta)$. In the case of odd-parity levels, only the lowest experimental values $\left(E<45000 \mathrm{~cm}^{-1}\right)$ published by Wyart (2010) were included in the fitting procedure using the $E_{\mathrm{av}}, F^{k}, G^{k}, R^{k}, \zeta_{n l}$ and $\alpha$ parameters of the $5 \mathrm{~d}^{4} 6 \mathrm{~s} 6 \mathrm{p}$, $5 \mathrm{~d}^{5} 6 \mathrm{p}$ and $5 \mathrm{~d}^{3} 6 \mathrm{~s}^{2} 6 \mathrm{p}$ configurations as variable parameters. The mean deviation, $|\Delta E|=\left|E_{\exp }-E_{\text {calc }}\right|$, obtained when fitting the even-parity levels ( 70 levels), was found to be equal to almost the same value in both models $\left(\left|\Delta E_{A}\right|=57 \mathrm{~cm}^{-1}\right.$ and $\left|\Delta E_{B}\right|=59 \mathrm{~cm}^{-1}$ ), while this parameter was slightly increased for odd-parity levels (141 levels) when going from model $\mathrm{A}$ to model $\mathrm{B}\left(\left|\Delta E_{A}\right|=64 \mathrm{~cm}^{-1}\right.$ and $\left|\Delta E_{B}\right|=$ $73 \mathrm{~cm}^{-1}$ ).

\section{Results and discussion}

The calculated energy levels and Landé $g$-factors are compared with experiment in tables 1 and 2 for even and odd parities, respectively. Detailed knowledge of the Landé factors is important to analyse the atomic spectra when an external magnetic field is applied. It can also provide us with useful information regarding the spin-orbit interaction and, consequently, the coupling schemes encountered in atoms. Moreover, the $g$-factor is helpful for the assignment of the energy levels in terms of analysis and allows us to get deeper insight into the properties of atomic states in heavy elements such as W I, for which this parameter was still unknown for many levels. When looking at tables 1 and 2, very good overall agreement is observed between the theoretical results (using both models A and B) and the available experimental Landé factors. The present determination of $g$-values in neutral tungsten is an extension of a similar work recently performed for some atoms and ions along the sixth row of the periodic table (Biémont et al 2010).

Calculated radiative lifetimes obtained in this work using model $\mathrm{A}, \tau_{A}$, and model $\mathrm{B}, \tau_{B}$, are compared to the available experimental measurements in table 2 for odd-parity levels below $45000 \mathrm{~cm}^{-1}$. This comparison is illustrated in figure 1 showing the ratio $\tau_{\exp } / \tau_{\text {calc }}$ as a function of the energy. It is seen that the overall agreement between experimental lifetimes and calculated ones is rather similar whatever the physical model used, the mean ratio $\tau_{\exp } / \tau_{\text {calc }}$ is found to be equal to $1.12 \pm 0.40$ and $1.02 \pm 0.38$ in the cases of models $\mathrm{A}$ and $\mathrm{B}$, respectively. We also note that the most important discrepancies with experimental measurements appear for some levels below $30000 \mathrm{~cm}^{-1}$ and above $40000 \mathrm{~cm}^{-1}$. This can be explained by the fact that the former levels, characterized by long lifetimes $\left(\tau_{\exp }>500 \mathrm{~ns}\right)$, are depopulated by weak transitions for which the computed line strengths were found to be affected by important cancellation effects, while the levels above $40000 \mathrm{~cm}^{-1}$ were found to be very strongly mixed, their eigenvector purities rarely exceeding $15 \%$. This is illustrated in figure 2 showing the $L S$-coupling purities obtained using 
Table 2. Experimental and calculated energies, Landé factors and radiative lifetimes for W I odd-parity levels below $45000 \mathrm{~cm}^{-1}$.

\begin{tabular}{|c|c|c|c|c|c|c|c|c|c|}
\hline$J$ & $\begin{array}{l}E_{\exp ^{\mathrm{a}}} \\
\left(\mathrm{cm}^{-1}\right)\end{array}$ & $\begin{array}{l}\Delta E_{A}{ }^{\mathrm{b}} \\
\left(\mathrm{cm}^{-1}\right)\end{array}$ & $\begin{array}{l}\Delta E_{B}{ }^{c} \\
\left(\mathrm{~cm}^{-1}\right)\end{array}$ & $g_{\exp }{ }^{\mathrm{a}}$ & $g_{A}^{\mathrm{b}}$ & $g_{B}^{\mathrm{c}}$ & $\begin{array}{l}\tau_{\exp }{ }^{d} \\
(\mathrm{~ns})\end{array}$ & $\begin{array}{l}\tau_{A}{ }^{b} \\
(\mathrm{~ns})\end{array}$ & $\begin{array}{l}\tau_{B}{ }^{\mathrm{c}} \\
\text { (ns) }\end{array}$ \\
\hline 0 & 19389.43 & -1 & 33 & & & & & 5817 & 5411 \\
\hline 1 & 20064.30 & 40 & 62 & 1.54 & 1.61 & 1.61 & $1880 \pm 140$ & 745 & 781 \\
\hline 2 & 21448.76 & -70 & -55 & 1.48 & 1.49 & 1.49 & & 1381 & 1469 \\
\hline 1 & 21453.90 & 14 & 23 & 2.51 & 2.45 & 2.45 & $275 \pm 14$ & 210 & 221 \\
\hline 3 & 23047.31 & -105 & -98 & 1.53 & 1.48 & 1.48 & & 1310 & 1432 \\
\hline 2 & 23964.67 & 7 & -8 & 1.93 & 1.90 & 1.90 & $250 \pm 13$ & 172 & 185 \\
\hline 4 & 24763.39 & -54 & -48 & 1.50 & 1.48 & 1.48 & $1800 \pm 180$ & 926 & 1017 \\
\hline 1 & 25983.60 & 143 & 72 & 0.54 & 0.49 & 0.49 & $860 \pm 43$ & 1134 & 933 \\
\hline 3 & 26189.20 & 45 & 2 & 1.80 & 1.74 & 1.74 & $161 \pm 8$ & 120 & 134 \\
\hline 2 & 26229.77 & -52 & -61 & 1.84 & 1.92 & 2.00 & $76.1 \pm 3.8$ & 78.2 & 83.8 \\
\hline 2 & 26367.28 & 200 & 226 & 0.87 & 0.81 & 0.72 & $315 \pm 16$ & 364 & 593 \\
\hline 0 & 26629.46 & -25 & 17 & & & & & 2738 & 3927 \\
\hline 5 & 26676.48 & 68 & 83 & 1.46 & 1.47 & 1.47 & $1230 \pm 80$ & 590 & 642 \\
\hline 3 & 27488.11 & 0 & -15 & 1.72 & 1.72 & 1.72 & $85.6 \pm 4.3$ & 91 & 102 \\
\hline 2 & 27662.52 & -56 & -77 & 1.21 & 1.22 & 1.23 & $182 \pm 9$ & 183 & 178 \\
\hline 1 & 27778.50 & -36 & -54 & 1.25 & 1.28 & 1.28 & $715 \pm 36$ & 846 & 892 \\
\hline 4 & 27889.68 & 74 & 52 & 1.71 & 1.68 & 1.68 & $59.4 \pm 1.2$ & 52.5 & 60.1 \\
\hline 1 & 28198.90 & -108 & -124 & & 2.22 & 2.25 & $132 \pm 7$ & 157 & 179 \\
\hline 4 & 28797.24 & 28 & 19 & 1.61 & 1.57 & 1.56 & $185 \pm 9$ & 186 & 205 \\
\hline 3 & 29139.12 & -3 & 43 & 1.06 & 1.06 & 1.07 & $257 \pm 13$ & 306 & 321 \\
\hline 2 & 29195.84 & -31 & -34 & 1.28 & 1.23 & 1.24 & $301 \pm 15$ & 453 & 490 \\
\hline 2 & 29393.40 & -75 & -101 & 1.83 & 1.79 & 1.78 & $71.4 \pm 3.6$ & 69.1 & 75.9 \\
\hline 6 & 29643.06 & 93 & 112 & & 1.48 & 1.48 & & 87290 & 102900 \\
\hline 5 & 29773.34 & 104 & 105 & 1.55 & 1.50 & 1.50 & $695 \pm 35$ & 467 & 473 \\
\hline 0 & 29823.09 & -26 & 63 & & & & & 844 & 969 \\
\hline 3 & 29912.85 & 12 & -16 & 1.31 & 1.35 & 1.36 & $168 \pm 8$ & 102 & 101 \\
\hline 3 & 30586.64 & 18 & -2 & 1.64 & 1.55 & 1.54 & $50.2 \pm 2.5$ & 55.2 & 60.6 \\
\hline 1 & 30683.54 & 13 & 81 & 1.39 & 1.42 & 1.39 & & 1108 & 1068 \\
\hline 1 & 31323.48 & 3 & -67 & 0.86 & 0.90 & 0.91 & $158 \pm 8$ & 170 & 171 \\
\hline 4 & 31432.91 & -121 & -84 & 1.32 & 1.31 & 1.30 & $449 \pm 22$ & 624 & 668 \\
\hline 2 & 31817.63 & 31 & 79 & 1.52 & 1.47 & 1.47 & & 619 & 767 \\
\hline 3 & 32238.02 & 7 & -25 & 1.3 & 1.42 & 1.42 & & 379 & 358 \\
\hline 0 & 32386.56 & -92 & -165 & & & & & 1289 & 1062 \\
\hline 4 & 32828.12 & 37 & 12 & 1.7 & 1.22 & 1.23 & & 610 & 546 \\
\hline 3 & 32957.58 & 24 & 92 & 1.43 & 1.41 & 1.41 & & 150 & 181 \\
\hline 2 & 33141.38 & 50 & 70 & 1.51 & 1.49 & 1.49 & $115 \pm 6$ & 97 & 103 \\
\hline 5 & 33370.04 & -41 & -11 & 1.39 & 1.38 & 1.37 & $439 \pm 22$ & 549 & 584 \\
\hline 2 & 33944.06 & 8 & -58 & & 1.19 & 1.19 & & 346 & 348 \\
\hline 4 & 34121.68 & -6 & -25 & 1.5 & 1.43 & 1.43 & $188 \pm 9$ & 219 & 205 \\
\hline 3 & 34228.60 & -21 & 12 & & 0.85 & 0.85 & & 836 & 898 \\
\hline 1 & 34342.44 & 90 & 123 & 1.56 & 1.56 & 1.55 & $89.9 \pm 2.1$ & 126 & 148 \\
\hline 3 & 34354.08 & -166 & -149 & 0.71 & 0.84 & 0.82 & $305 \pm 15$ & 270 & 287 \\
\hline 2 & 34485.86 & -126 & -165 & 0.82 & 0.90 & 0.88 & $65.9 \pm 3.3$ & 119 & 157 \\
\hline 4 & 34632.60 & 71 & 82 & 0.89 & 0.90 & 0.90 & & 396 & 395 \\
\hline 1 & 34719.33 & -126 & -249 & 0.15 & 0.26 & 0.27 & $22.1 \pm 0.5$ & 24.2 & 24.1 \\
\hline 4 & 35116.78 & -84 & -63 & 1.2 & 1.21 & 1.21 & $83.9 \pm 4.2$ & 72.4 & 83.8 \\
\hline 2 & 35311.56 & -121 & -128 & 1.0 & 0.74 & 0.74 & & 108 & 96 \\
\hline 3 & 35499.15 & -37 & -70 & 1.0 & 1.11 & 1.12 & $166 \pm 8$ & 226 & 272 \\
\hline 5 & 35507.07 & -29 & -63 & & 1.17 & 1.16 & & 855 & 822 \\
\hline 2 & 35731.96 & -68 & -127 & 1.5 & 1.53 & 1.52 & $25.7 \pm 1.3$ & 19.0 & 20.7 \\
\hline 3 & 35943.17 & 33 & 31 & 1.4 & 1.28 & 1.27 & $282 \pm 14$ & 229 & 271 \\
\hline 4 & 36082.30 & 1 & 23 & 1.24 & 1.25 & 1.25 & $57.9 \pm 2.9$ & 52.5 & 53.5 \\
\hline 1 & 36190.49 & 124 & 105 & 1.62 & 1.62 & 1.60 & $18.37 \pm 0.38$ & 14.8 & 17.4 \\
\hline 5 & 36275.10 & 43 & 78 & 1.27 & 1.21 & 1.22 & & 180 & 171 \\
\hline 0 & 36588.32 & -71 & -104 & & & & & 37.6 & 38.1 \\
\hline 2 & 36673.70 & 43 & 82 & 1.50 & 1.63 & 1.81 & & 16.8 & 10.4 \\
\hline 3 & 36874.36 & 140 & 118 & 1.50 & 1.52 & 1.53 & $10.95 \pm 0.23$ & 8.3 & 8.8 \\
\hline 2 & 36904.16 & 19 & 60 & 1.57 & 1.53 & 1.42 & $8.55 \pm 0.18$ & 7.3 & 12.1 \\
\hline 4 & 37146.36 & -22 & -21 & 1.1 & 1.06 & 1.05 & $89.0 \pm 4.4$ & 92 & 108 \\
\hline 5 & 37309.16 & -25 & -12 & 1.25 & 1.25 & 1.23 & $42.4 \pm 2.1$ & 37.2 & 48.7 \\
\hline 6 & 37451.33 & -30 & -29 & & 1.19 & 1.18 & & 524 & 573 \\
\hline 2 & 37466.30 & -164 & -187 & 1.28 & 1.17 & 1.14 & & 10.3 & 13.1 \\
\hline 3 & 37674.08 & 63 & -3 & 1.13 & 1.13 & 1.11 & & 26.1 & 33.8 \\
\hline 1 & 37773.96 & -155 & -51 & & 0.62 & 0.64 & & 15.9 & 17.7 \\
\hline
\end{tabular}


Table 2. (Continued.)

\begin{tabular}{|c|c|c|c|c|c|c|c|c|c|}
\hline$J$ & $\begin{array}{l}E_{\exp }{ }^{\mathrm{a}} \\
\left(\mathrm{cm}^{-1}\right)\end{array}$ & $\begin{array}{l}\Delta E_{A}{ }^{\mathrm{b}} \\
\left(\mathrm{cm}^{-1}\right)\end{array}$ & $\begin{array}{l}\Delta E_{B} \mathrm{c} \\
\left(\mathrm{cm}^{-1}\right)\end{array}$ & $g_{\exp }{ }^{\mathrm{a}}$ & $g_{A}^{\mathrm{b}}$ & $g_{B}{ }^{\mathrm{c}}$ & $\begin{array}{l}\tau_{\exp }{ }^{d} \\
(n s)\end{array}$ & $\begin{array}{l}\tau_{A}{ }^{b} \\
(\mathrm{~ns})\end{array}$ & $\begin{array}{l}\tau_{B}{ }^{c} \\
(\mathrm{~ns})\end{array}$ \\
\hline 4 & 38001.12 & -70 & -107 & 1.1 & 1.16 & 1.17 & $111 \pm 6$ & 115 & 134 \\
\hline 3 & 38053.05 & 59 & 126 & 1.11 & 1.20 & 1.24 & & 14.7 & 14.6 \\
\hline 6 & 38203.12 & 145 & 180 & & 1.31 & 1.32 & & 951 & 1182 \\
\hline 3 & 38206.38 & -57 & -132 & & 0.93 & 0.91 & & 25.8 & 34.0 \\
\hline 4 & 38259.40 & -47 & -15 & & 1.27 & 1.28 & $16.44 \pm 0.36$ & 13.3 & 13.6 \\
\hline 1 & 38355.84 & 104 & -58 & & 1.95 & 1.82 & $20.0 \pm 0.5$ & 14.2 & 11.7 \\
\hline 0 & 38576.14 & -46 & -26 & & & & & 146 & 170 \\
\hline 4 & 38748.44 & 43 & 16 & & 1.04 & 1.03 & & 22.0 & 27.1 \\
\hline 2 & 39030.25 & -45 & -51 & & 1.18 & 1.14 & $15.80 \pm 0.34$ & 14.2 & 18.6 \\
\hline 1 & 39183.20 & 179 & 188 & 1.01 & 1.11 & 1.31 & $4.44 \pm 0.10$ & 3.7 & 5.2 \\
\hline 5 & 39361.01 & 84 & 108 & 1.13 & 1.11 & 1.11 & $81.7 \pm 4.0$ & 75.5 & 78.8 \\
\hline 5 & 39614.05 & -10 & 4 & 1.20 & 1.14 & 1.14 & & 46.4 & 56.7 \\
\hline 1 & 39636.62 & 229 & 254 & 1.44 & 1.22 & 1.14 & $11.74 \pm 0.25$ & 12.1 & 12.0 \\
\hline 3 & 39646.41 & -26 & -42 & 1.46 & 1.47 & 1.35 & $8.11 \pm 0.21$ & 7.3 & 10.3 \\
\hline 2 & 39707.02 & -7 & -8 & 1.00 & 1.14 & 1.09 & & 24.3 & 33.1 \\
\hline 7 & 39709.04 & 29 & 54 & & 1.24 & 1.24 & & 393 & 485 \\
\hline 4 & 39719.96 & 13 & 24 & 1.17 & 1.09 & 1.11 & $19.72 \pm 0.76$ & 25.1 & 22.6 \\
\hline 2 & 40011.50 & -41 & -69 & 1.00 & 1.0 & 0.99 & $15.48 \pm 0.34$ & 14.7 & 16.6 \\
\hline 4 & 40233.97 & 44 & 18 & 1.53 & 1.22 & 1.23 & $11.67 \pm 0.29$ & 14.7 & 16.4 \\
\hline 3 & 40269.35 & 288 & 316 & 1.03 & 0.98 & 1.04 & & 32.1 & 26.8 \\
\hline 1 & 40411.12 & 32 & -4 & 1.58 & 1.43 & 1.41 & $6.85 \pm 0.15$ & 5.9 & 7.1 \\
\hline 5 & 40476.42 & -56 & -58 & 1.04 & 1.16 & 1.17 & & 65.5 & 67.5 \\
\hline 4 & 40583.07 & 6 & -19 & & 1.35 & 1.32 & $11.81 \pm 0.27$ & 5.5 & 7.2 \\
\hline 3 & 40665.85 & 72 & 46 & 0.96 & 1.00 & 1.00 & $46.1 \pm 1.0$ & 26.5 & 32.2 \\
\hline 1 & 40770.78 & 15 & 51 & 1.28 & 1.38 & 1.43 & $8.09 \pm 0.19$ & 8.1 & 8.5 \\
\hline 2 & 40868.40 & 12 & -18 & 1.26 & 1.31 & 1.34 & $7.71 \pm 0.17$ & 4.7 & 5.5 \\
\hline 5 & 40911.98 & 5 & -16 & 1.03 & 1.14 & 1.14 & & 96 & 102 \\
\hline 3 & 40923.83 & 35 & 13 & 1.32 & 1.28 & 1.28 & & 18.1 & 22.3 \\
\hline 2 & 41104.52 & 31 & -8 & 1.5 & 1.50 & 1.46 & & 20.3 & 24.4 \\
\hline 0 & 41127.38 & 77 & 149 & & & & $10.25 \pm 0.23$ & 17.1 & 13.4 \\
\hline 6 & 41171.44 & 151 & 162 & & 1.23 & 1.23 & & 146 & 161 \\
\hline 4 & 41198.14 & -68 & -36 & 1.22 & 1.25 & 1.24 & & 12.6 & 14.3 \\
\hline 6 & 41417.52 & -26 & 41 & 1.23 & 1.15 & 1.14 & & 386 & 455 \\
\hline 3 & 41499.43 & 23 & -37 & 1.11 & 1.19 & 1.22 & $13.58 \pm 0.41$ & 10.2 & 10.6 \\
\hline 2 & 41583.20 & -41 & 35 & 1.06 & 1.38 & 1.43 & $8.53 \pm 0.20$ & 5.5 & 6.6 \\
\hline 3 & 41694.34 & -72 & -94 & 1.28 & 1.29 & 1.29 & $14.39 \pm 0.34$ & 7.8 & 8.4 \\
\hline 2 & 41734.13 & 46 & 99 & 1.1 & 1.02 & 1.08 & $12.61 \pm 0.28$ & 6.3 & 5.7 \\
\hline 4 & 41871.94 & 8 & 48 & 1.11 & 1.06 & 1.05 & & 45.5 & 60.7 \\
\hline 0 & 41965.24 & -6 & -109 & & & & & 5.7 & 6.7 \\
\hline 2 & 41978.62 & 135 & 120 & 0.8 & 0.83 & 0.84 & $12.29 \pm 0.31$ & 39.3 & 69.1 \\
\hline 6 & 42239.06 & 82 & 89 & & 1.13 & 1.13 & & 253 & 306 \\
\hline 3 & 42251.51 & 82 & 26 & 1.32 & 1.22 & 1.23 & $8.28 \pm 0.18$ & 11.2 & 11.5 \\
\hline 1 & 42262.30 & 129 & 94 & 1.5 & 1.51 & 1.41 & & 17.4 & 13.0 \\
\hline 2 & 42449.60 & -227 & -258 & & 1.21 & 1.21 & & 11.4 & 11.9 \\
\hline 4 & 42450.24 & 76 & 142 & & 1.00 & 1.01 & & 56.3 & 63.1 \\
\hline 3 & 42514.14 & 40 & 28 & 1.22 & 1.15 & 1.18 & & 23.8 & 35.5 \\
\hline 4 & 42532.62 & 96 & 38 & & 1.08 & 1.08 & & 40.6 & 36.6 \\
\hline 1 & 42573.49 & -23 & 35 & 1.3 & 1.00 & 1.08 & $3.81 \pm 0.11$ & 3.4 & 4.0 \\
\hline 3 & 42601.19 & -37 & -12 & 1.12 & 1.14 & 1.09 & $15.17 \pm 0.34$ & 20.1 & 21.8 \\
\hline 5 & 42866.00 & 119 & 89 & 1.11 & 1.15 & 1.14 & & 54.2 & 61.6 \\
\hline 4 & 42910.74 & -105 & -170 & 1.18 & 1.22 & 1.21 & & 13.3 & 14.3 \\
\hline 5 & 43034.10 & -14 & -73 & & 1.14 & 1.14 & $39.6 \pm 0.8$ & 42.9 & 44.8 \\
\hline 0 & 43053.88 & -202 & -160 & & & & & 2.9 & 3.4 \\
\hline 1 & 43217.33 & 17 & 25 & 1.3 & 0.99 & 1.00 & $9.58 \pm 0.20$ & 13.2 & 13.9 \\
\hline 2 & 43227.66 & 58 & 61 & 1.3 & 1.04 & 1.06 & $7.62 \pm 0.16$ & 8.8 & 9.7 \\
\hline 4 & 43251.00 & 28 & -74 & 1.14 & 1.19 & 1.16 & $14.37 \pm 0.29$ & 16.9 & 23.3 \\
\hline 7 & 43411.50 & -171 & -200 & 1.20 & 1.21 & 1.21 & & 170 & 220 \\
\hline 3 & 43478.58 & 96 & 83 & 1.3 & 1.18 & 1.16 & & 11.1 & 14.0 \\
\hline 2 & 43514.68 & -128 & -90 & 0.9 & 0.95 & 0.92 & & 20.3 & 21.0 \\
\hline 4 & 43720.87 & 12 & 57 & & 1.13 & 1.11 & & 37.4 & 32.6 \\
\hline 5 & 43741.37 & 44 & -21 & 1.09 & 1.11 & 1.11 & & 94.1 & 93.2 \\
\hline 3 & 43850.84 & -106 & -72 & 1.17 & 1.07 & 1.11 & $7.80 \pm 0.20$ & 6.5 & 7.3 \\
\hline 1 & 43892.62 & 80 & 101 & 1.05 & 1.39 & 1.40 & $6.57 \pm 0.24$ & 5.6 & 6.2 \\
\hline 5 & 43924.25 & -34 & -32 & 1.2 & 1.09 & 1.09 & & 108 & 111 \\
\hline
\end{tabular}


Table 2. (Continued.)

\begin{tabular}{llcccccccc}
\hline & $\begin{array}{l}E_{\exp }{ }^{\mathrm{a}} \\
\left(\mathrm{cm}^{-1}\right)\end{array}$ & $\begin{array}{c}\Delta E_{A}{ }^{\mathrm{b}} \\
\left(\mathrm{cm}^{-1}\right)\end{array}$ & $\begin{array}{c}\Delta E_{B}{ }^{\mathrm{c}} \\
\left(\mathrm{cm}^{-1}\right)\end{array}$ & $g_{\exp }{ }^{\mathrm{a}}$ & $g_{A}{ }^{\mathrm{b}}$ & $g_{B}{ }^{\mathrm{c}}$ & $\begin{array}{l}\tau_{\exp }{ }^{\mathrm{d}} \\
(\mathrm{ns})\end{array}$ & $\begin{array}{l}\tau_{A}{ }^{\mathrm{b}} \\
(\mathrm{ns})\end{array}$ & $\begin{array}{l}\tau_{B}{ }^{\mathrm{c}} \\
(\mathrm{ns})\end{array}$ \\
\hline 2 & 43975.22 & -19 & 38 & 1.15 & 1.19 & 1.21 & $4.06 \pm 0.12$ & 6.0 & 4.8 \\
4 & 43985.41 & -57 & 2 & 1.24 & 1.12 & 1.13 & & 21.7 & 22.1 \\
3 & 44021.00 & -77 & -117 & 1.2 & 1.24 & 1.24 & $10.82 \pm 0.22$ & 9.8 & 9.0 \\
1 & 44353.46 & -27 & 67 & 1.02 & 1.35 & 1.32 & & 4.5 & 5.7 \\
2 & 44367.50 & -13 & 57 & 1.1 & 1.10 & 1.03 & & 9.3 & 13.2 \\
6 & 44390.42 & 55 & 55 & 1.28 & 1.15 & 1.14 & & 96 & 116 \\
3 & 44447.02 & -58 & -75 & 1.38 & 1.21 & 1.21 & $8.31 \pm 0.19$ & 7.2 & 6.9 \\
5 & 44546.76 & -145 & -129 & 1.3 & 1.20 & 1.17 & & 14.0 & 18.2 \\
2 & 44596.28 & 53 & 43 & 1.11 & 1.37 & 1.39 & & 10.0 & 18.7 \\
1 & 44737.21 & -51 & 103 & & 0.90 & 0.92 & & 10.2 & 9.6 \\
6 & 44923.90 & -31 & -19 & & 1.20 & 1.20 & & 30.0 & 29.6 \\
4 & 44940.57 & -92 & -36 & & 1.11 & 1.12 & & 24.0 & 24.6 \\
7 & 44970.82 & 82 & 80 & & 1.20 & 1.20 & & 75.9 & 77.1 \\
\hline
\end{tabular}

a From Wyart (2010).

b Present work-model A $\left(\Delta E_{A}=E_{\text {exp }}-E_{A}\right)$.

${ }^{\mathrm{c}}$ Present work-model B $\left(\Delta E_{B}=E_{\text {exp }}-E_{B}\right)$.

${ }^{\mathrm{d}}$ From Den Hartog et al (1987) and Schnabel and Kock (1997).

Table 3. Radiative transition probabilities for atomic tungsten lines. Only the most intense transitions $\left(\mathrm{gA}_{\mathrm{calc}} \geqslant 5 \times 10^{7} \mathrm{~s}^{-1}\right)$ are listed in the table.

\begin{tabular}{|c|c|c|c|c|c|c|c|c|c|}
\hline $\begin{array}{l}\lambda^{\mathrm{a}} \\
(\mathrm{nm})\end{array}$ & $\begin{array}{c}E_{\text {Low }}{ }^{\mathrm{b}} \\
\left(\mathrm{cm}^{-1}\right)\end{array}$ & $J$ & $\begin{array}{c}E_{\mathrm{Upp} .}^{\mathrm{b}} \\
\left(\mathrm{cm}^{-1}\right)\end{array}$ & $J^{\prime}$ & Int. ${ }^{b}$ & $\begin{array}{c}g A_{\exp 1}{ }^{c} \\
\left(10^{8} \mathrm{~s}^{-1}\right)\end{array}$ & $\begin{array}{l}g A_{\exp 2}{ }^{\mathrm{d}} \\
\left(10^{8} \mathrm{~s}^{-1}\right)^{-1}\end{array}$ & $\begin{array}{r}g A_{A}{ }^{\mathrm{e}} \\
\left(10^{8} \mathrm{~s}^{-1}\right)\end{array}$ & $\begin{array}{r}g A_{B}{ }^{\mathrm{f}} \\
\left(10^{8} \mathrm{~s}^{-1}\right)\end{array}$ \\
\hline 225.391 & 0.00 & 0 & 44353.46 & 1 & 25 & & & 1.18 & 0.95 \\
\hline 227.7583 & 0.00 & 0 & 43892.62 & 1 & 60 & & $1.86(0.21)$ & 2.38 & 2.10 \\
\hline 231.3170 & 0.00 & 0 & 43217.33 & 1 & 30 & & $1.47(0.18)$ & 1.09 & 0.97 \\
\hline 232.8872 & 1670.29 & 1 & 44596.28 & 2 & 15 & & & 0.77 & 0.23 \\
\hline 236.3072 & 1670.29 & 1 & 43975.22 & 2 & 18 & & $3.45(0.35)$ & 3.75 & 3.58 \\
\hline 236.7693 & 1670.29 & 1 & 43892.62 & 1 & 10 & & $0.70(0.09)$ & 0.79 & 0.62 \\
\hline 238.9078 & 1670.29 & 1 & 43514.68 & 2 & 15 & & & 0.75 & 0.16 \\
\hline 240.5580 & 1670.29 & 1 & 43227.66 & 2 & 30 & & $3.45(0.35)$ & 2.79 & 2.67 \\
\hline 241.4039 & 3325.53 & 2 & 44737.21 & 1 & 20 & & & 0.79 & 0.99 \\
\hline 241.5685 & 1670.29 & 1 & 43053.88 & 0 & 35 & & & 3.36 & 2.82 \\
\hline 242.2292 & 3325.53 & 2 & 44596.28 & 2 & 30 & & & 1.65 & 0.50 \\
\hline 243.1081 & 3325.53 & 2 & 44447.02 & 3 & 25 & & $2.01(0.21)$ & 3.72 & 6.01 \\
\hline 243.5782 & 3325.53 & 2 & 44367.50 & 2 & 12 & & & 1.89 & 1.03 \\
\hline 243.6624 & 3325.53 & 2 & 44353.46 & 1 & 25 & & & 4.02 & 2.94 \\
\hline 244.4056 & 1670.29 & 1 & 42573.49 & 1 & 60 & & $2.62(0.16)$ & 3.01 & 2.18 \\
\hline 245.1484 & 1670.29 & 1 & 42449.60 & 2 & 70 & & & 2.86 & 2.77 \\
\hline 245.1996 & 0.00 & 0 & 40770.78 & 1 & 100 & & $2.34(0.23)$ & 1.16 & 0.91 \\
\hline 245.6534 & 3325.53 & 2 & 44021.00 & 3 & 150 & & $3.30(0.28)$ & 1.43 & 1.10 \\
\hline 245.9300 & 3325.53 & 2 & 43975.22 & 2 & 140 & & $4.31(0.43)$ & 2.66 & 3.65 \\
\hline 246.2793 & 1670.29 & 1 & 42262.30 & 1 & 100 & & & 0.97 & 1.45 \\
\hline 246.4305 & 3325.53 & 2 & 43892.62 & 1 & 60 & & $1.29(0.15)$ & 1.41 & 1.17 \\
\hline 246.6848 & 3325.53 & 2 & 43850.84 & 3 & 100 & & $4.52(0.35)$ & 7.38 & 6.53 \\
\hline 248.0130 & 1670.29 & 1 & 41978.62 & 2 & 100 & & $2.35(0.25)$ & 0.59 & 0.04 \\
\hline 248.0955 & 1670.29 & 1 & 41965.24 & 0 & 60 & & & 1.55 & 1.32 \\
\hline 248.9720 & 3325.53 & 2 & 43478.58 & 3 & 35 & & & 4.29 & 3.38 \\
\hline 249.5264 & 1670.29 & 1 & 41734.13 & 2 & 100 & & $2.33(0.23)$ & 3.20 & 4.70 \\
\hline 250.4698 & 1670.29 & 1 & 41583.20 & 2 & 60 & & $1.58(0.18)$ & 4.80 & 3.41 \\
\hline 251.3934 & 4830.00 & 3 & 44596.28 & 2 & 30 & & & 1.17 & 0.65 \\
\hline 252.3410 & 4830.00 & 3 & 44447.02 & 3 & 60 & & $3.59(0.39)$ & 2.24 & 1.11 \\
\hline 252.8486 & 4830.00 & 3 & 44367.50 & 2 & 50 & & & 2.80 & 1.94 \\
\hline 253.3635 & 1670.29 & 1 & 41127.38 & 0 & 30 & & $0.98(0.07)$ & 0.52 & 0.70 \\
\hline 254.5340 & 3325.53 & 2 & 42601.19 & 3 & 50 & & $1.69(0.14)$ & 1.19 & 1.05 \\
\hline 254.7136 & 3325.53 & 2 & 42573.49 & 1 & 100 & & $5.25(0.30)$ & 4.61 & 4.49 \\
\hline 255.0378 & 1670.29 & 1 & 40868.40 & 2 & 60 & & $1.40(0.15)$ & 2.19 & 1.44 \\
\hline 255.0843 & 4830.00 & 3 & 44021.00 & 3 & 4 & & & 3.16 & 4.67 \\
\hline 255.100 & 3325.53 & 2 & 42514.14 & 3 & 25 & & & 0.78 & 0.42 \\
\hline 255.1349 & 0.00 & 0 & 39183.20 & 1 & 200 & & $5.34(0.45)$ & 7.51 & 5.44 \\
\hline 255.3824 & 4830.00 & 3 & 43975.22 & 2 & 100 & & $2.13(0.20)$ & 0.92 & 2.35 \\
\hline 255.5207 & 3325.53 & 2 & 42449.60 & 2 & 40 & & & 0.66 & 0.58 \\
\hline
\end{tabular}


Table 3. (Continued.)

\begin{tabular}{|c|c|c|c|c|c|c|c|c|c|}
\hline $\begin{array}{l}\lambda^{\mathrm{a}} \\
(\mathrm{nm})\end{array}$ & $\begin{array}{c}E_{\text {Low }}{ }^{b} \\
\left(\mathrm{~cm}^{-1}\right)\end{array}$ & $J$ & $\begin{array}{c}E_{\mathrm{Upp} .}^{\mathrm{b}} \\
\left(\mathrm{cm}^{-1}\right)\end{array}$ & $J^{\prime}$ & Int. $^{b}$ & $\begin{array}{r}g A_{\exp 1}{ }^{\mathrm{c}} \\
\left(10^{8} \mathrm{~s}^{-1}\right)\end{array}$ & $\begin{aligned} g A_{\exp 2}{ }^{\mathrm{d}} & \left(10^{8} \mathrm{~s}^{-1}\right)\end{aligned}$ & $\begin{array}{r}g A_{A} \mathrm{e}^{-} \\
\left(10^{8} \mathrm{~s}^{-1}\right)\end{array}$ & $\begin{array}{r}g A_{B}{ }^{\mathrm{f}} \\
\left(10^{8} \mathrm{~s}^{-1}\right)\end{array}$ \\
\hline 255.6749 & 1670.29 & 1 & 40770.78 & 1 & 40 & & $0.79(0.08)$ & 1.81 & 1.93 \\
\hline 256.1968 & 4830.00 & 3 & 43850.84 & 3 & 100 & & $3.35(0.28)$ & 0.95 & 0.32 \\
\hline 256.8214 & 3325.53 & 2 & 42251.51 & 3 & 30 & & $0.23(0.02)$ & 0.61 & 0.74 \\
\hline 258.0337 & 2951.29 & 3 & 41694.34 & 3 & 30 & & $0.73(0.11)$ & 1.86 & 1.77 \\
\hline 258.0487 & 1670.29 & 1 & 40411.12 & 1 & 125 & & $2.25(0.15)$ & 3.37 & 2.81 \\
\hline 260.2804 & 3325.53 & 2 & 41734.13 & 2 & 25 & & $0.47(0.05)$ & 3.29 & 2.75 \\
\hline 260.3544 & 4830.00 & 3 & 43227.66 & 2 & 40 & & $1.41(0.15)$ & 1.81 & 1.64 \\
\hline 260.6388 & 0.00 & 0 & 38355.84 & 1 & 35 & & & 1.63 & 2.15 \\
\hline 260.7378 & 1670.29 & 1 & 40011.50 & 2 & 35 & & $0.60(0.04)$ & 1.01 & 0.99 \\
\hline 260.832 & 6219.33 & 4 & 44546.76 & 5 & 50 & & & 3.71 & 2.54 \\
\hline 261.3076 & 3325.53 & 2 & 41583.20 & 2 & 80 & & $2.70(0.30)$ & 0.55 & 0.84 \\
\hline 261.3818 & 2951.29 & 3 & 41198.14 & 4 & 50 & & & 3.85 & 3.29 \\
\hline 261.5124 & 6219.33 & 4 & 44447.02 & 3 & 40 & & $1.11(0.13)$ & 1.68 & 1.26 \\
\hline 262.0215 & 2951.29 & 3 & 41104.52 & 2 & 50 & & & 0.90 & 0.64 \\
\hline 262.5220 & 4830.00 & 3 & 42910.74 & 4 & 60 & & & 3.72 & 3.01 \\
\hline 262.8255 & 1670.29 & 1 & 39707.02 & 2 & 40 & & & 0.90 & 0.67 \\
\hline 263.2695 & 2951.29 & 3 & 40923.83 & 3 & 80 & & & 2.13 & 1.81 \\
\hline 263.3129 & 1670.29 & 1 & 39636.62 & 1 & 150 & & $1.96(0.15)$ & 1.66 & 1.54 \\
\hline 264.6185 & 3325.53 & 2 & 41104.52 & 2 & 70 & & & 1.26 & 1.10 \\
\hline 264.6536 & 0.00 & 0 & 37773.96 & 1 & 15 & & & 0.54 & 0.59 \\
\hline 264.6730 & 4830.00 & 3 & 42601.19 & 3 & 80 & & $1.08(0.08)$ & 0.65 & 0.50 \\
\hline 264.7091 & 6219.33 & 4 & 43985.41 & 4 & 60 & & & 0.89 & 1.02 \\
\hline 265.1546 & 4830.00 & 3 & 42532.62 & 4 & 5 & & & 0.80 & 0.90 \\
\hline 265.2844 & 4830.00 & 3 & 42514.14 & 3 & 8 & & & 0.89 & 0.63 \\
\hline 265.6540 & 2951.29 & 3 & 40583.07 & 4 & 300 & & $6.07(0.41)$ & 15.90 & 12.10 \\
\hline 265.7361 & 4830.00 & 3 & 42450.24 & 4 & 60 & & & 1.14 & 0.94 \\
\hline 266.2835 & 3325.53 & 2 & 40868.40 & 2 & 200 & & $2.11(0.15)$ & 5.00 & 4.21 \\
\hline 267.1472 & 4830.00 & 3 & 42251.51 & 3 & 200 & & $2.74(0.25)$ & 3.60 & 3.40 \\
\hline 267.7276 & 3325.53 & 2 & 40665.85 & 3 & 100 & & $1.11(0.08)$ & 1.60 & 1.31 \\
\hline 267.8878 & 2951.29 & 3 & 40269.35 & 3 & 150 & & & 0.61 & 1.18 \\
\hline 268.1422 & 2951.29 & 3 & 40233.97 & 4 & 400 & & $6.62(0.45)$ & 5.16 & 4.66 \\
\hline 269.567 & 3325.53 & 2 & 40411.12 & 1 & 150 & & $1.41(0.12)$ & 0.94 & 0.80 \\
\hline 269.9594 & 6219.33 & 4 & 43251.00 & 4 & 150 & & $3.42(0.27)$ & 2.64 & 1.32 \\
\hline 270.8927 & 4830.00 & 3 & 41734.13 & 2 & 80 & & & 0.71 & 0.78 \\
\hline 271.1846 & 4830.00 & 3 & 41694.34 & 3 & 8 & & & 0.85 & 0.81 \\
\hline 271.5503 & 6219.33 & 4 & 43034.10 & 5 & 75 & & $1.53(0.11)$ & 0.76 & 0.96 \\
\hline 271.8906 & 2951.29 & 3 & 39719.96 & 4 & 250 & & $4.26(0.27)$ & 3.11 & 3.47 \\
\hline 272.0046 & 4830.00 & 3 & 41583.20 & 2 & 30 & & & 1.74 & 1.18 \\
\hline 272.4352 & 2951.29 & 3 & 39646.41 & 3 & 300 & & $7.35(0.56)$ & 8.74 & 5.86 \\
\hline 272.4624 & 6219.33 & 4 & 42910.74 & 4 & 35 & & & 1.85 & 2.56 \\
\hline 272.7954 & 6219.33 & 4 & 42866.00 & 5 & 40 & & & 1.30 & 1.12 \\
\hline 274.8844 & 4830.00 & 3 & 41198.14 & 4 & 80 & & & 2.42 & 2.30 \\
\hline 276.8982 & 1670.29 & 1 & 37773.96 & 1 & 100 & & & 1.21 & 0.98 \\
\hline 276.9741 & 4830.00 & 3 & 40923.83 & 3 & 80 & & & 0.93 & 0.76 \\
\hline 277.0880 & 2951.29 & 3 & 39030.25 & 2 & 200 & & $0.92(0.08)$ & 1.32 & 0.97 \\
\hline 277.3999 & 4830.00 & 3 & 40868.40 & 2 & 200 & & $1.96(0.15)$ & 2.03 & 2.24 \\
\hline 277.4476 & 6219.33 & 4 & 42251.51 & 3 & 300 & & $3.33(0.28)$ & 1.14 & 1.10 \\
\hline 279.2696 & 2951.29 & 3 & 38748.44 & 4 & 60 & & & 1.57 & 1.17 \\
\hline 279.9928 & 3325.53 & 2 & 39030.25 & 2 & 50 & & $0.65(0.05)$ & 0.62 & 0.51 \\
\hline 281.8060 & 6219.33 & 4 & 41694.34 & 3 & 250 & & $3.02(0.28)$ & 4.43 & 3.91 \\
\hline 283.1379 & 2951.29 & 3 & 38259.40 & 4 & 250 & & $4.42(0.36)$ & 5.91 & 5.41 \\
\hline 283.3630 & 6219.33 & 4 & 41499.43 & 3 & 120 & & $3.35(0.25)$ & 4.21 & 4.16 \\
\hline 283.9340 & 9528.06 & 0 & 44737.21 & 1 & 50 & & & 0.79 & 0.78 \\
\hline 284.1570 & 4830.00 & 3 & 40011.50 & 2 & 80 & & $0.87(0.06)$ & 1.01 & 0.90 \\
\hline 284.8022 & 2951.29 & 3 & 38053.05 & 3 & 180 & & & 2.98 & 2.91 \\
\hline 285.6030 & 1670.29 & 1 & 36674.08 & 2 & 150 & & & 0.92 & 0.32 \\
\hline 286.6062 & 3325.53 & 2 & 38206.38 & 3 & 200 & & & 1.60 & 1.18 \\
\hline 287.8721 & 3325.53 & 2 & 38053.05 & 3 & 50 & & & 1.08 & 1.31 \\
\hline 287.9112 & 2951.29 & 3 & 37674.08 & 3 & 150 & & & 1.46 & 1.04 \\
\hline 287.9396 & 0.00 & 0 & 34719.33 & 1 & 140 & $0.73(0.09)$ & $0.74(0.05)$ & 0.91 & 0.95 \\
\hline 288.1606 & 6219.33 & 4 & 40911.98 & 5 & 80 & & & 0.69 & 0.71 \\
\hline 289.6009 & 1670.29 & 1 & 36190.49 & 1 & 150 & & $0.84(0.05)$ & 0.85 & 0.77 \\
\hline 289.6442 & 2951.29 & 3 & 37466.30 & 2 & 400 & & & 4.04 & 3.09 \\
\hline 291.8254 & 6219.33 & 4 & 40476.42 & 5 & 100 & & & 1.12 & 1.17 \\
\hline
\end{tabular}


Table 3. (Continued.)

\begin{tabular}{|c|c|c|c|c|c|c|c|c|c|}
\hline $\begin{array}{l}\lambda^{\mathrm{a}} \\
(\mathrm{nm})\end{array}$ & $\begin{array}{c}E_{\text {Low }}{ }^{b} \\
\left(\mathrm{~cm}^{-1}\right)\end{array}$ & $J$ & $\begin{array}{c}E_{\mathrm{Upp} .}^{\mathrm{b}} \\
\left(\mathrm{cm}^{-1}\right)\end{array}$ & $J^{\prime}$ & Int. ${ }^{b}$ & $\begin{array}{r}g A_{\exp 1}{ }^{\mathrm{c}} \\
\left(10^{8} \mathrm{~s}^{-1}\right)\end{array}$ & $\begin{array}{r}g A_{\exp 2}{ }^{\mathrm{d}} \\
\left(10^{8} \mathrm{~s}^{-1}\right)\end{array}$ & $\begin{array}{r}g A_{A}^{\mathrm{e}} \\
\left(10^{8} \mathrm{~s}^{-1}\right)\end{array}$ & $\begin{array}{r}g A_{B}{ }^{\mathrm{f}} \\
\left(10^{8} \mathrm{~s}^{-1}\right)\end{array}$ \\
\hline 292.3103 & 4830.00 & 3 & 39030.25 & 2 & 150 & & $0.38(0.03)$ & 0.50 & 0.33 \\
\hline 293.4996 & 1670.29 & 1 & 35731.96 & 2 & 250 & $0.73(0.08)$ & & 0.97 & 0.93 \\
\hline 294.4398 & 2951.29 & 3 & 36904.16 & 2 & 300 & & $5.40(0.40)$ & 6.24 & 3.00 \\
\hline 294.6989 & 2951.29 & 3 & 36874.36 & 3 & 300 & & $5.76(0.42)$ & 7.01 & 6.69 \\
\hline 294.7388 & 4830.00 & 3 & 38748.44 & 4 & 150 & & & 2.03 & 1.79 \\
\hline 296.4520 & 2951.29 & 3 & 36673.70 & 2 & 150 & & & 1.77 & 4.36 \\
\hline 297.9860 & 3325.53 & 2 & 36874.36 & 3 & 70 & & & 1.08 & 0.96 \\
\hline 299.3614 & 6219.33 & 4 & 39614.05 & 5 & 125 & & & 1.96 & 1.54 \\
\hline 301.3788 & 4830.00 & 3 & 38001.12 & 4 & 120 & $0.58(0.04)$ & & 0.51 & 0.20 \\
\hline 301.6466 & 6219.33 & 4 & 39361.01 & 5 & 150 & $1.02(0.06)$ & & 0.86 & 0.88 \\
\hline 301.7436 & 2951.29 & 3 & 36082.30 & 4 & 200 & $1.09(0.07)$ & & 1.18 & 1.29 \\
\hline 302.5263 & 9528.06 & 0 & 42573.49 & 1 & 50 & & & 0.64 & 0.46 \\
\hline 304.1863 & 3325.53 & 2 & 36190.49 & 1 & 80 & & & 0.63 & 0.53 \\
\hline 304.9688 & 2951.29 & 3 & 35731.96 & 2 & 120 & $0.86(0.07)$ & & 1.28 & 1.12 \\
\hline 321.5562 & 6219.33 & 4 & 37309.16 & 5 & 200 & $2.30(0.10)$ & & 2.70 & 2.04 \\
\hline 330.0822 & 4830.00 & 3 & 35116.78 & 4 & 150 & $0.73(0.05)$ & & 0.73 & 0.57 \\
\hline 339.1520 & 15069.93 & 5 & 44546.76 & 5 & 80 & & & 0.52 & 0.53 \\
\hline 353.7446 & 15460.01 & 3 & 43720.87 & 4 & 80 & & & 0.53 & 0.58 \\
\hline 357.5220 & 17008.50 & 6 & 44970.82 & 7 & 80 & & & 1.24 & 1.15 \\
\hline 361.7515 & 2951.29 & 3 & 30586.64 & 3 & 500 & $0.75(0.04)$ & & 0.65 & 0.59 \\
\hline 365.0997 & 17008.50 & 6 & 44390.42 & 6 & 60 & & & 0.61 & 0.56 \\
\hline 377.3706 & 13777.71 & 2 & 40269.35 & 3 & 150 & & & 0.68 & 0.59 \\
\hline 381.0380 & 18116.84 & 2 & 44353.46 & 1 & 100 & & & 0.61 & 0.51 \\
\hline 394.7977 & 19648.54 & 6 & 44970.82 & 7 & 20 & & & 0.69 & 0.76 \\
\hline 395.5304 & 19648.54 & 6 & 44923.90 & 6 & 40 & & & 1.16 & 1.09 \\
\hline 396.8590 & 19256.24 & 4 & 44447.02 & 3 & 20 & & $0.65(0.07)$ & 0.83 & 0.62 \\
\hline 398.3292 & 19826.04 & 5 & 44923.90 & 6 & 50 & & & 2.88 & 2.97 \\
\hline 400.8753 & 2951.29 & 3 & 27889.68 & 4 & 1000 & $1.47(0.07)$ & $1.50(0.11)$ & 1.65 & 1.43 \\
\hline 401.5218 & 19648.54 & 6 & 44546.76 & 5 & 100 & & & 2.46 & 1.76 \\
\hline 403.6855 & 19256.24 & 4 & 44021.00 & 3 & 40 & & $1.04(0.07)$ & 0.85 & 0.42 \\
\hline 407.4358 & 2951.29 & 3 & 27488.11 & 3 & 600 & $0.73(0.04)$ & & 0.68 & 0.61 \\
\hline 411.8052 & 18974.51 & 3 & 43251.00 & 4 & 25 & & $0.83(0.06)$ & 0.51 & 0.25 \\
\hline 413.8018 & 19826.04 & 5 & 43985.41 & 4 & 40 & & & 0.60 & 0.51 \\
\hline 420.4405 & 19256.24 & 4 & 43034.10 & 5 & 30 & & $0.57(0.04)$ & 0.98 & 0.83 \\
\hline 420.7052 & 19648.54 & 6 & 43411.50 & 7 & 50 & & & 0.77 & 0.60 \\
\hline 427.5486 & 18116.84 & 2 & 41499.43 & 3 & 50 & & $0.65(0.05)$ & 0.79 & 0.78 \\
\hline 429.4606 & 2951.29 & 3 & 26229.77 & 2 & 800 & $0.62(0.03)$ & $0.62(0.02)$ & 0.61 & 0.56 \\
\hline
\end{tabular}

\footnotetext{
a Observed air wavelengths taken from Kramida and Shirai (2006).

b From Kramida and Shirai (2006).

c From Den Hartog et al (1987). Absolute uncertainties are given in parentheses.

${ }^{\mathrm{d}}$ From Kling and Kock (1999). Absolute uncertainties are given in parentheses.

e Present work-model A.

${ }^{\mathrm{f}}$ Present work-model B.

$A(B)$ stands for $A \times 10^{B}$.
}

theoretical model A for all of the odd-parity levels below $45000 \mathrm{~cm}^{-1}$.

The calculated transition probabilities, $g A$, obtained in this work using both models $\mathrm{A}$ and $\mathrm{B}$ are reported in table 3 alongside the lower (even) and upper (odd) experimental energy levels of the transition and the air wavelengths in $\mathrm{nm}$. These wavelengths were taken from the compilation of Kramida and Shirai (2006). Only transitions with calculated $g A$-values (model A) greater than $5 \times 10^{7} \mathrm{~s}^{-1}$ are listed in table 3. The complete table is available in our DESIRE database at the following address: http://w3.umons.ac.be/ astro/desire.shtml. Experimental transition probabilities, when available, are also given in table 3 for comparison. These values were obtained by Den Hartog et al (1987) and Kling and Kock (1999). When looking at this table, we note that the general agreement between theoretical results and experimental ones is better in the case of model $\mathrm{A}$ than in the case of model $\mathrm{B}$, the mean ratio $g A_{\exp } / g A_{\text {calc }}$ is found to be equal to $1.10 \pm$ 0.74 and $1.36 \pm 1.32$, respectively, if we except the line at $248.0130 \mathrm{~nm}$ for which the calculated transition probability is affected by large cancellation effects in model B. When compared with experimental $g A$-values, results of calculation $\mathrm{B}$ also seem to show a larger systematic underestimate and a larger scattering than those deduced from calculation A. This is probably due to the fact that core-valence interactions are better represented in model A than in model B. Consequently, it is expected that transition probabilities obtained in this work using model $\mathrm{A}$ are more reliable than those computed with 

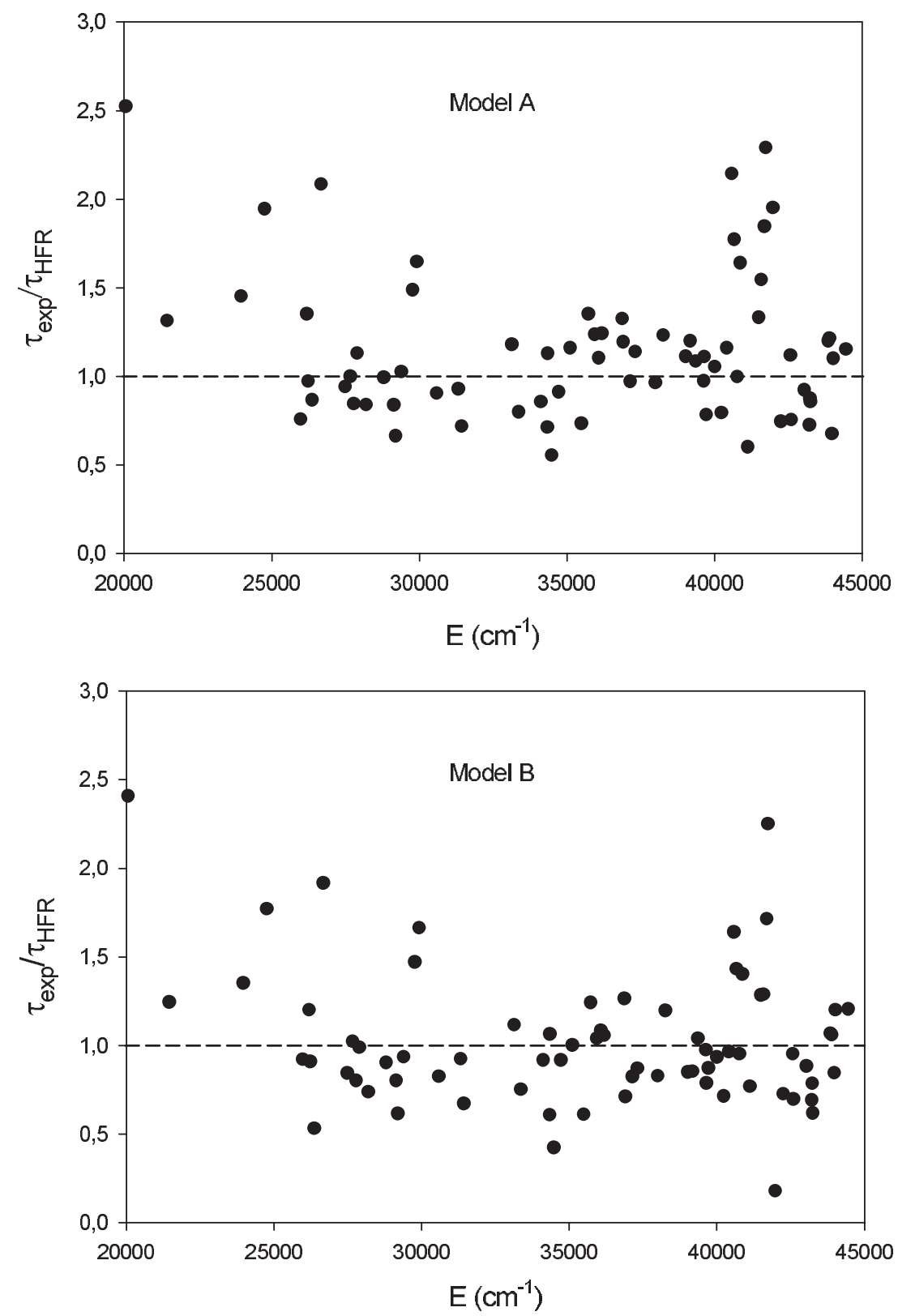

Figure 1. Comparison between calculated radiative lifetimes as obtained in this work using both physical models (A and B) and experimental values published by Den Hartog et al (1987) and Schnabel and Kock (1997).

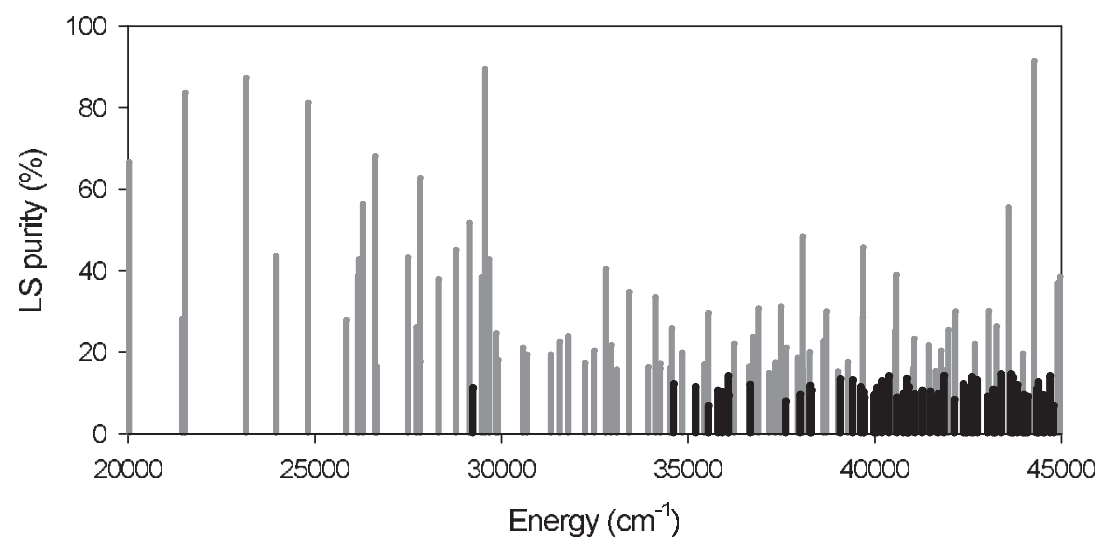

Figure 2. Wavefunction purities (in $L S$ coupling) for W I odd-parity levels below $45000 \mathrm{~cm}^{-1}$. The bold lines represent level purities smaller than $15 \%$. 


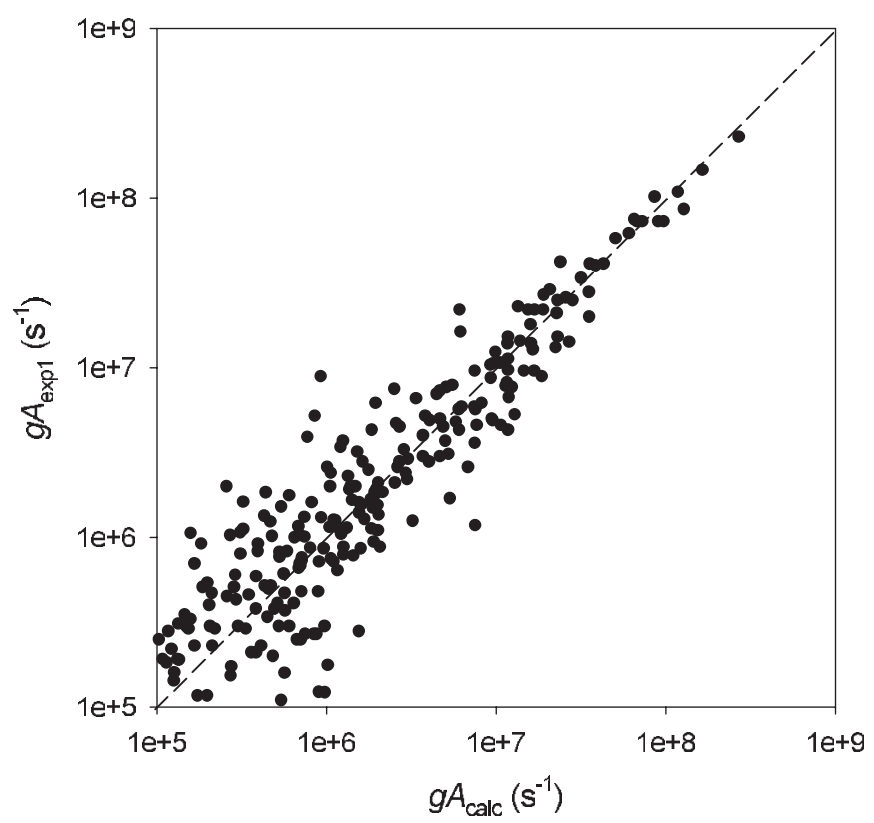

Figure 3. Comparison between calculated transition probabilities $\left(g A_{\text {calc }}\right)$ as obtained in this work (model $\left.\mathrm{A}\right)$ and experimental values $\left(g A_{\exp 1}\right)$ published by Den Hartog et al (1987).

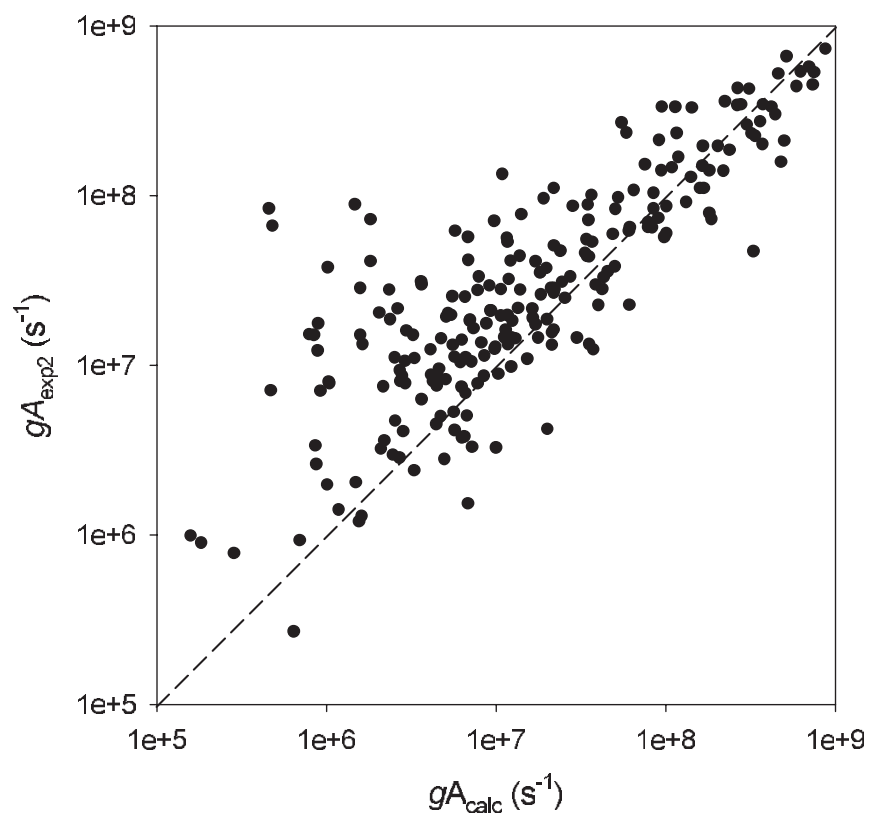

Figure 4. Comparison between calculated transition probabilities $\left(g A_{\text {calc }}\right)$ as obtained in this work (model $\left.\mathrm{A}\right)$ and experimental values ( $\left.g A_{\exp 2}\right)$ published by Kling and Kock (1999).

model B. This statement should however be checked on a firmer basis by new experimental measurements.

It is also worth noting that better overall agreement is observed when comparing the calculations with the experimental transition probabilities measured by Den Hartog et al (1987) than with those reported by Kling and Kock (1999), as shown in figures 3 and 4, a larger scattering being observed in the latter case even for intense transitions. This is probably due to the fact that the work of Kling and Kock was focused on higher lying levels of which most are characterized by very strong mixings in the wavefunction expansions, as already mentioned above for levels with $E>40000 \mathrm{~cm}^{-1}$.

\section{Conclusion}

A new set of computed transition probabilities for neutral tungsten lines is reported in this work. The accuracy of these results was assessed through detailed comparisons between two different HFR models and available experimental data. These data represent the first complete set of spectroscopic parameters computed for the very complex W I atomic structure. It is expected that the results will provide plasma physicists with some of the data they need for spectroscopic diagnostics and modelling of fusion plasmas magnetically confined in reactors where tungsten is expected to be used as a facing material.

\section{Acknowledgments}

PQ, PP and EB are, respectively, senior research associate, research associate and research director of the FRSFNRS. Financial support from the ADAS-EU project is acknowledged.

\section{References}

Biémont E, Palmeri P and Quinet P 2010 J. Phys. B: At. Mol. Opt. Phys. 43074010

Campbell-Miller M D and Simard B 1996 J. Opt. Soc. Am. B 132115

Clawson J E and Miller M H 1973 J. Opt. Soc. Am. 631598

Corliss C H 1969 J. Res. Natl Bur. Stand. A 73277

Corliss C H and Bozman W R 1962 National Bureau of Standards Monograph 53 (Washington, DC: US Government Printing Office)

Cowan R D 1981 The Theory of Atomic Structure and Spectra (Berkeley, CA: University of California Press)

Den Hartog E A, Duquette D W and Lawler J E 1987 J. Opt. Soc. Am. B 448

Duquette D W, Salih S and Lawler J E 1981 Phys. Rev. A 242847

Federici G et al 2001 Nucl. Fusion 411967

Fraga S, Karwowski J and Saxena K M S 1976 Handbook of Atomic Data (Amsterdam: Elsevier)

Kling R and Kock M 1999 J. Quant. Spectrosc. Radiat. Transfer 62129

Kramida A E and Shirai T 2006 J. Phys. Chem. Ref. Data 35423

Kwiatkowski M, Micali G, Werner K, Schmidt M and Zimmermann P 1982 Z. Phys. A 304197

Laun D D and Corliss C H 1968 J. Res. Natl Bur. Stand. A 72609

Martin W C, Zalubas R and Hagan L 1978 National Bureau of Standards Monograph 60 (Washington, DC: US Department of Commerce)

Naujoks D et al (ASDEX Upgrade Team) 1996 Nucl. Fusion 36671

Nilsson H, Engström L, Lundberg H, Palmeri P, Fivet V, Quinet P and Biémont É 2008 Eur. Phys. J. D 4913

Obbarius H U and Kock M 1982 J. Phys. B: At. Mol. Phys. 15527

Palmeri P, Quinet P, Fivet V, Biémont E, Nilsson H, Engström L and Lundberg H 2008 Phys. Scr. 78015304

Plekhotkin G A and Verolainen Ya F 1985 Opt. Spectrosc. 58447

Pospieszczyk A 2006 Nuclear Fusion Research (Berlin: Springer) 
Quinet P, Palmeri P, Biémont E, McCurdy M M, Rieger G,

Pinnington E H, Wickliffe M E and Lawler J E 1999 Mon. Not. R. Astron. Soc. 307934

Quinet P, Vinogradoff V, Palmeri P and Biémont E 2010 J. Phys. B: At. Mol. Opt. Phys. 43144003
Schnabel R and Kock M 1997 Z. Phys. D 4131

Shadmi Y and Caspi E 1968 J. Res. Natl Bur. Stand. A 72757 Skinner H C 2008 Can. J. Phys. 86285

Wyart J-F 1978 Phys. Scr. 1887

Wyart J-F 2010 J. Phys. B: At. Mol. Opt. Phys. 43074018 